\title{
Chapter 16 \\ Negative Binomial GAM and GAMM to Analyse Amphibian Roadkills
}

\author{
A.F. Zuur, A. Mira, F. Carvalho, E.N. Ieno, A.A. Saveliev, G.M. Smith, \\ and N.J. Walker
}

\subsection{Introduction}

This chapter analyses amphibian fatalities along a road in Portugal. The data are counts of kills making a Gaussian distribution unlikely; restricting our choice of techniques. We began with generalised linear models (GLM) and generalised additive models (GAM) with a Poisson distribution, but these models were overdispersed. To solve this, you can either apply a quasi-Poisson GLM or GAM, or use the negative binomial distribution (Chapter 9). In this particular example, either approaches can be applied as the overdispersion was fairly small (around 5), but with many ecological data sets it can be considerably larger, in which case the negative binomial GLM (or GAM) is the natural choice. As many textbooks give examples using quasi-Poisson GAMs and GLMs and only a few using the negative binomial, we decided to use the negative binomial distribution.

We chose GAM because the relationships between roadkills and explanatory variables were non-linear. We address issues like collinearity, residual patterns, and spatial correlations.

\subsubsection{Roadkills}

Since the second part of the twentieth century, roads have become a common feature in contemporary landscapes. For example, in North America alone, the road network has reached eight million kilometres and road construction is still increasing. Roads provide people and goods mobility, and are a central element in society (Forman et al., 2002). However, their impact on wildlife can be harmful as they (i) fragment populations, (ii) present barriers to dispersal as well as access to food and mates, and (iii) restrict gene flow. Also a large numbers of fatalities can occur as a result of animal-vehicle collisions.

A.F. Zuur $(\bowtie)$

Highland Statistics Ltd., Newburgh, AB41 6FN, United Kingdom 